Acta Hispanica (2015) 20: 121-130

\title{
EL USO DE LOS SUJETOS PRONOMINALES EXPLÍCITOS Y NULOS POR LOS APRENDICES DE ELE
}

\author{
RĂZVAN BRAN
}

Universidad de Bucarest

\begin{abstract}
Resumen
Este trabajo se propone analizar los errores cometidos por los estudiantes rumanos que aprenden el español como L2 en cuanto al uso de los sujetos pronominales explícitos y nulos. $\mathrm{Ni}$ el rumano (L1 de los estudiantes), ni el español requieren necesariamente el uso del sujeto explícito, pero tras investigar el corpus (formado por ensayos argumentativos y textos narrativos), hemos notado el uso inadecuado de los sujetos pronominales. En nuestra opinión, este error sintáctico se explica por la interferencia con otros idiomas aprendidos por los estudiantes, que requieren sujetos explícitos. Este tipo de error aparece con frecuencia en la interlengua inicial y disminuye con el nivel lingüístico adquirido. Como la competencia sintáctica es fundamental en el proceso de aprendizaje de la L2, es importantísimo que el fenómeno reciba más atención en las clases de ELE.
\end{abstract}

Palabras clave: sujeto pronominal, sujeto nulo, errores, interferencia, ELE

\begin{abstract}
This paper aims to analyse the errors made by Romanian students who learn Spanish as a Foreign Language in using pronominal and null subjects. Neither Romanian (students' L1) nor Spanish require pro subjects, but after investigating the corpus (including opinion essays and narratives), we have noticed that pronominal subjects are misused. In our view, one possible explanation of this syntactic error could be the interference with other foreign languages that students learn, which are pro-drop languages. This type of error appears more often in the first stage of interlingua and the more advanced the student is, the less errors he makes. As the syntactic competence is fundamental in the acquisition process of the $\mathrm{L} 2$, it is very important this phenomenon to be reckoned both by students and by teachers.
\end{abstract}

Key words: subject pronouns, pro drop subject, errors, interference, Spanish as a Foreign Language.

\section{Introducción}

La sintaxis desempeña un papel fundamental en el aprendizaje de una lengua extranjera, puesto que además de la adquisición de las reglas fonológicas y morfológicas, para producir un mensaje coherente, tanto escrito, como oral, es imprescindible que los hablantes desarrollen la competencia sintáctica. Las unidades léxicas deben agruparse para 
El uso de los sujetos pronominales explícitos y nulos por los aprendices de ELE

formar oraciones con sentido, correctas desde el punto de vista sintáctico, para transmitir el mensaje adecuado. Por ejemplo, los aprendices han de adquirir no solo los vocablos de una lengua y su sentido o la morfología nominal y verbal, sino también las reglas que permiten construir enunciados correctos sintácticamente. Por ello, el sujeto, una de las funciones sintácticas básicas, necesita una atención especial por parte de los aprendices, siempre de acuerdo con las particularidades de cada lengua.

En el presente estudio se analizará el uso de los sujetos pronominales por los estudiantes rumanos que aprenden el español como lengua extranjera. Se trata de principiantes e intermedios, es decir los niveles A1-B1 según el Marco Común Europeo de Referencia. La investigación se apoya en un corpus formado por más de cien redacciones y tiene como objetivos identificar los errores sintácticos que afectan al sujeto e intentar explicarlos. Al final, se proponen unas soluciones para mejorar el nivel de español en cuanto a la competencia sintáctica de los estudiantes no nativos (rumanos) y corregir los errores que afectan al uso de sujeto pronominal y nulo.

\section{Consideraciones generales sobre el sujeto en español}

El sujeto es uno de los argumentos esenciales del verbo y una de las funciones sintácticas fundamentales. Además, desempeña un papel comunicativo muy importante, ya que el sujeto se identifica con el tema. El español, como la mayoría de las lenguas romances, permite oraciones con sujetos explícitos y nulos (pro). Hay idiomas, tales como el inglés o el francés, en que la presencia del sujeto explícito (en forma de palabras léxicas o gramaticales) es obligatoria por la pérdida de flexión verbal. La falta de flexión del verbo puede conducir a la ambigüedad y, por lo tanto, las razones que no permiten la omisión del sujeto son de índole pragmática y comunicativa. Frente a esos idiomas, en español el sujeto (fonético) puede omitirse en la estructura superficial, en unas cuantas situaciones que no afectan la transmisión del sentido ${ }^{1}$. Si el lector o el oyente pueden interpretar correctamente la información recibida del contexto, entonces la presencia del sujeto no es

\footnotetext{
${ }^{1}$ Pablo DEVÍS MÁRQUEZ en su artículo "El Parámetro del Sujeto Nulo y la enseñanza del español como lengua extranjera. Reflexión gramatical”, in: Didáctica. Lengua y Literatura, Vol. 23, Madrid, 2011, 59-86, pasa revista todas las situaciones en las que se puede omitir el sujeto en castellano. Otros estudio concerniente al tema es el de BOSQUE, I., "Clases de sujetos tácitos", in: Borrego Nieto, J. - Gómez Asencio, J. J. - Santos Río, L. (eds.), Philologica II. Homenaje a D. Antonio Llorente, Salamanca, Universidad de Salamanca, 1989, 91-111; Per ROSENGREN, "Presencia y ausencia de los pronombres personales sujetos en español moderno", in: Acta Universitatis Gothoburgensis, Estocolmo, 1974. También, hay investigaciones contrastivas que analizan la presencia obligatoria del sujeto explícito en español y otros idiomas, como el de Carmen MUÑOZ, "La presencia obligatoria del sujeto pronominal en inglés y en castellano", in: Atlantis, vol. X, 1988, 37-44; Juana M. LICERAS y Lourdes DÍAZ: "Topic drop versus pro drop: null subjects and pronominal subjects in the Spanish L of Chinese English French German and Japanese speakers", in: Second language research, 15-1, 1999, 1-40.
} 


\section{Răzvan Bran}

obligatoria. Además de las desinencias que indican persona y número, el conocimiento de la situación y del contexto explica cuál es el sujeto del verbo.

No obstante, hay situaciones en las que el sujeto explícito es obligatorio, cuando el hablante quiere enfatizar el sujeto o hacer un contraste entre varias entidades (sujetos), como en los ejemplos que se dan a continuación en (1a-b):

(1) a. Juan y yo tuvimos que entregar nuestros trabajos ayer. Yo lo entregué, pero él todavía no.

b. El que no tiene dinero soy yo, no ella.

En (1), los sujetos en negrilla tienen valor enfático y el sujeto pronominal explícito marca la oposición entre dos entidades de la realidad extralingüística.

Por otro lado, las situaciones más frecuentes en que el sujeto léxico o pronominal se omite son las siguientes. Primero, si hay correferencialidad de sujetos o el sujeto del verbo está bajo control a través de otro elemento sintáctico de la oración (sujeto, objeto directo o indirecto, posesivos):

(2) a. Juani no sabe protocari el piano.

b. proNo puedo procomer.

En (2a) hay dos verbos: saber en modo personal (indicativo, tercera persona, singular), con sujeto expreso, y tocar en modo no personal (infinitivo). El primer verbo tiene sujeto expreso, pero el segundo, que va en infinitivo, tiene un sujeto controlado por el sujeto Juan del verbo regente. Además, el infinitivo no puede construirse con un sujeto explícito. En (2b), estamos ante una oración con dos verbos: no puedo, conjugado en modo indicativo (primera persona, singular) y comer, en infinitivo. El predicado no puedo tiene sujeto gramatical (yo omitido). Aquí el uso del pronombre de primera persona no es obligatorio, ya que las marcas morfológicas verbales indican cuál es el sujeto. Por otro lado, entre los sujetos de los dos verbos se establece una relación de identidad y, por consiguiente, el sujeto del infinitivo está bajo control, como en (2a). También, emplear el pronombre sujeto con el verbo en infinitivo sería imposible y resultaría una oración agramatical. Por lo tanto, la oración (2b) es perfectamente gramatical y el interlocutor entiende quién hace la acción.

En segundo lugar, se permite el empleo del sujeto tácito si éste es recuperable del contexto:

(3) a. Juan no está en casa. proEstá de vacaciones.

b. Miguel viene, porque prono se siente bien.

Tanto en (3a), como en (3b), los sujetos de los predicados está y no se siente son tácitos, pero recuperables del contexto, dado que remiten a Juan y a Miguel, mencionados 
El uso de los sujetos pronominales explícitos y nulos por los aprendices de ELE

anteriormente. El sujeto de la segunda oración es correferencial con el de la primera, por ello, no hace falta volver a expresarlo. El contexto es bastante claro para que el interlocutor interprete correctamente el mensaje.

Además, tanto en rumano, como en español, el sistema verbal expresa las categorías morfológicas (número, persona, pero no la de género) mediante las desinencias personales, que aclaran la forma verbal y permite al interlocutor identificar el argumento elidido

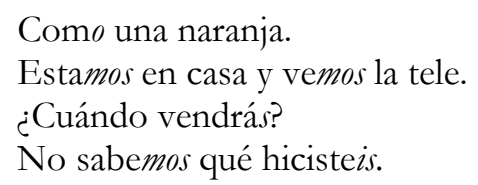

En los ejemplos anteriores, de (4), el uso de los sujetos pronominales expresos no es necesario, ya que las marcas morfológicas de los verbos en primera o segunda persona (los así llamados sujetos gramaticales) indican claramente la persona: el locutor y el interlocutor. Las situaciones en que los morfemas gramaticales de número y persona son morfos cero o representan un caso de ambigüedad morfológica y semántica no son poco frecuentes, pero, las más de las veces, el contexto situacional proporciona la suficiente información sobre el sujeto del verbo. Sólo en ocasiones pueden producirse confusiones referenciales entre la primera persona, singular, y la tercera, singular, por ejemplo, en el pretérito imperfecto de indicativo (venía), el condicional (vendría, habría venido), el subjuntivo (venga, viniera, haya venido, hubiera / bubiese venido).

El sujeto (explícito pronominal o nulo) establece relaciones anafóricas con elementos ya mencionados en el contexto $^{3}$. Esto significa que el sujeto remite a elementos anteriores, llamados antecedentes, lo que permite identificar la entidad de la realidad extralingüística que realiza la acción del verbo.

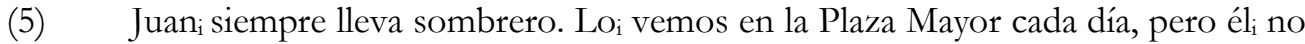
nos ha visto nunca.

En el ejemplo (5), el sujeto pronominal él establece una relación anafórica y lógica con el complemento directo lo y éste, a su vez, con el sujeto léxico Juan, mencionado

\footnotetext{
${ }^{2}$ Sin embargo, hay lenguas que no permiten la elisión del sujeto explícito a pesar de su rica flexión verbal (p. ej. el alemán y el islandés) y lenguas de sujeto nulo (pro-drop), que permiten sujetos nulos, que no tienen una riqueza en cuanto a la flexión del verbo (p. ej. el chino y el japonés).

3 Susana PERALES, María Rosario PORTILlO MAYORGA, "Sobre las propiedades referenciales de los sujetos nulos y pronominales del español oral y escrito", in: Las destrezas orales en la enseñanza del español L2-LE: XVII Congreso Internacional de la Asociación del Español como lengua extranjera (ASELE), Vol. 2, Logroño, 2007, 889-900.
} 
anteriormente. Por ello, el sujeto tácito o el sujeto expresado mediante los pronombres personales o demostrativos son recuperables del contexto ${ }^{4}$.

Opinamos que, en el caso de la adquisición de una lengua pro drop, el tema del sujeto es muy importante. También, cabe mencionar que hasta ahora no se han llevado a cabo estudios en este ámbito concernientes a los aprendices rumanos. El sujeto nulo es un rasgo común que comparten el español y el rumano, puesto que en la estructura superficial la presencia del sujeto explícito no es obligatoria. Por consiguiente, partimos de la hipótesis de que los aprendices rumanos de ELE no cometen errores en el uso de los sujetos pronominales y nulos, dado que se ha demostrado que el Parámetro del Sujeto Nulo se aprende bastante pronto incluso por los aprendices de ELE cuya lengua materna es el inglés (una lengua de sujeto explícito obligatorio) ${ }^{5}$. Nuestra investigación se apoya en el estudio de P. Pablo Devís Márquez ${ }^{6}$, por un lado, y en el de Susana Perales y Rosario Portillo ${ }^{7}$, por otro lado. Nos interesan la producción y la omisión de los sujetos pronominales, vistas desde la perspectiva de las relaciones anafóricas que se establecen entre dichos sujetos y sus antecedentes.

Asimismo, nuestro trabajo tiene un propósito didáctico, que concierne al proceso de enseñanza-aprendizaje del español como lengua segunda, e intenta proponer unas soluciones metodológicas para corregir el uso inadecuado del sujeto.

Devís Márquez afirma que el fenómeno de la supresión del sujeto ha recibido poca atención en la literatura de especialidad y, por eso, este problema no parece que haya sido aclarado, al menos en lo que se refiere al aprendizaje del español L2 ${ }^{8}$. Es más, hay autores que afirman que el uso de los sujetos explícitos y nulos no es un asunto de máxima importancia en el aprendizaje de ELE, ya que se pueden adquirir sólo por inmersión en el input de la L29.

\section{El corpus}

Los datos escritos que hemos analizado provienen de redacciones (textos narrativos y ensayos argumentativos), es decir pruebas de producción espontánea, que pertenecen a estudiantes de distintos cursos (primer, segundo y tercer curso). Los aprendices estudian español como lengua extranjera en la Facultad de Lenguas y Literaturas Extranjeras

\footnotetext{
${ }^{4}$ Los pronombres personales, los demostrativos y algunos de los adverbios pertenecen a la clase de los deícticos, es decir, son elementos que están relacionados con el contexto y cambian su función anafórica según el contexto.

${ }^{5}$ Cristóbal LOZANO, "Knowledge of Expletive and Pronominal Subjects by Learners of Spanish", in: ITL Review of Applied Linguistics, 13, 2002, 37-60.

${ }^{6}$ DEVÍS MÁRQUEZ, op. cit., 59-86.

7 PERALES, PORTILLO MAYORGA, op. cit.

${ }^{8}$ DEVÍS MÁRQUEZ, op. cit., 59-86.

${ }^{9}$ Carlos SOLER MONTES, "Principios y parámetros en la adquisición del español como lengua extranjera: el parámetro del sujeto nulo", in: Cuadernos Cervantes de la Lengua Española 8, Madrid, 39, 2002, 33-36.
} 
El uso de los sujetos pronominales explícitos y nulos por los aprendices de ELE

(Universidad de Bucarest) - Filología y Lenguas Modernas Aplicadas. El corpus de este trabajo está formado por más de cien redacciones. Los estudiantes tienen cuatro clases de lengua española y estudian tanto gramática (norma y uso), como comunicación (escrita, oral, comprensión lectora). Además del español, la mayoría de los aprendices estudian también inglés, francés o rumano. Cabe mencionar que no hemos corregido los fragmentos escritos por los estudiantes y, por lo tanto, pueden aparecer otros casos de agramaticalidad que conciernen a la ortografía, morfología, sintaxis, semántica etc. Para iniciar el análisis de los errores, reproducimos más abajo unos fragmentos recogidos de las redacciones mencionadas en líneas anteriores. Reproducimos a continuación unos fragmentos sólo a modo de ejemplo, dado que los casos ilustrados son los más frecuentes y representativos en el corpus analizado.

(i) Todos estos casos pasan en los tiempos. Porque si nosotros nos referimos a tiempos pasados [...], esto no sería el caso.

(ii) Hoy en día la publicidad está tomando muchas formas. Ella puede ser de dos tipos.

(iii) Hoy día la publicidad es una cosa muy importante para la gente y también para las empresas, porque ella ayuda a la promovación.

(iv) Desde mucho tiempo se habla de publicidad y cada día ella es cambiada, mejor dicho, cada día es modernizada y trae nuevas ideas.

(v) Por un lado, la publicidad es benéfica, porque ella muestra lo que es bueno.

(vi) Todos los días nos encontramos con ella, en la calle, en la televisión, en internet aún si nosotros no la queremos ver.

(vii) Algunas semanas antes, este verano, yo fui miembro de una Organización No Gubernamental.

(viii) Hoy día las campañas publicitarias son frecuentes, porque estas son difusadas en la tele y también en internet.

(ix) Esta es mi amiga María. Ella llega al público a través de los medios de comunicación.

(x) Cada día hablan por teléfono y se visitan cada fin de semana. Ellas se conocen de hace mucho tiempo, cuando mi madre era muy joven y era estudiante en la Universidad.

(xi) ¿Puedo decir a Stefy tu nombre entero para que ella vea si su madre te conoce?

(xii) Juan era un niño que vivía muy feliz con su madre, María, en una pequeña casa. Éliba a la escuela todos los días y era un chico muy inteligente.

(xiii) Juan no sabía qué hacer, pero él creía que su mami estaba bromeando.

(xiv) Así que le gusta decir que yo soy su hija porque somos muy parecidas.

$(x v)$ Los adultos no tienen muchos amigos, porque ellos nunca juegan y no tienen juguetes.

(xvi) Mi compañera Paula dice que ella tiene mucho más amigos que yo, porque tiene dos perritos y una gatita (pero ellos son malos y huyen de los padres de Paula). 
Răzvan Bran

\section{Análisis de errores}

En este apartado presentaremos los usos indebidos de los pronombres en función de sujeto. Los errores más frecuentes cometidos por los estudiantes rumanos se refieren al uso impropio del sujeto explícito pronominal no sólo cuando tiene valor enfático o cuando es necesario marcar una oposición, un contraste:

(6) El pastel lo he comido yo, no él.

En (6), los pronombres desempeñan una función contrastiva y se utilizan para enfatizar o desambiguar un cierto contexto de la enunciación. Por lo tanto, tienen una función más bien pragmática y no sintáctica. Muchas veces, aunque el sujeto ya ha sido mencionado en el contexto más amplio del enunciado o del discurso, los aprendices tienden a marcarlo de nuevo. Este es el caso de las construcciones adversativas (xiii, $x v i)$, de las proposiciones subordinadas causales (iii, $v$, viii, $x v)$ o finales $(x i)$, pero también en oraciones principales, regentes o no. Otras veces, el uso enfático del pronombre sujeto no se explica, como en $(i, v i, v i i)$.

En cuanto a las propiedades referenciales de los sujetos pronominales mencionados en los fragmentos de arriba, los pronombres en función de sujeto se usan con valor anafórico y se refieren a un sujeto anterior, como en (ii, iii, $v$, viii, ix, $x, x i i, x v i)$. Sólo en pocos casos $(i v, x i)$ el sujeto pronominal hace referencia a un complemento. De esta manera, se puede decir que el uso de los sujetos por los rumanos no está en concordancia con los resultados obtenidos por Perales y Portillo Mayorga ${ }^{10}$. La adquisición y la gramaticalidad de los sujetos pronominales y / o nulos se hace desde el punto de vista de la producción, a saber, si los enunciados producidos por los aprendices se asemejan a los que producen los hablantes nativos. Así, en el estudio ya mencionado, las autoras llegaron a la conclusión de que los nativos españoles utilizan los sujetos pronominales con referencia a un complemento, y no a antecedente en función de sujeto. Por lo tanto, desde el punto de vista de la referencia, los aprendices rumanos del español no respetan las reglas de producción propias a la lengua española.

Del material lingüístico analizado se puede desprender la conclusión de que el uso inadecuado de los sujetos pronominales (explícitos) disminuye con el nivel de lengua adquirido por los estudiantes. El uso abusivo de sujetos explícitos aparece en la interlengua inicial, es decir, los principiantes tienden a cometer más errores con el sujeto mientras que, en los textos escritos por los que tienen un nivel medio de español, los sujetos pronominales agramaticales son poco frecuentes. Por lo tanto, el sujeto nulo es una competencia sintáctica que se adquiere con el pasar del tiempo y con el mejoramiento general de los conocimientos lingüísticos (morfosintaxis, vocabulario).

${ }^{10}$ PERALES, PORTILLO MAYORGA, op. cit., 889-900. 
El uso de los sujetos pronominales explícitos y nulos por los aprendices de ELE

Depende en gran medida de la información de entrada (input) que reciben y procesan los aprendices, es decir de la cantidad de información, textos audio o escritos con los que entran en contacto.

En segundo lugar, cabe notar que se trata de una influencia de los idiomas en que la presencia del sujeto pronominal es obligatoria. Los estudiantes que tienen como lengua materna el rumano tienden a utilizar el sujeto a pesar de que no es necesario. Se podría considerar que en el caso de los rumanos no debería ocurrir ese tipo de error sintáctico, dado que tanto el rumano como el español comparten el rasgo de los sujetos pro drop. En nuestra opinión, se trata de un caso interesante de interferencia lingüística, pero no con la lengua materna, sino con los demás idiomas que el estudiante aprende o ha aprendido. En Rumanía, las dos lenguas extranjeras más aprendidas son el inglés y el francés, que requieren la presencia de un sujeto explícito (pronominal o léxico). Por consiguiente, los aprendices del español L2, acostumbrados al uso obligatorio del sujeto, tienden a extender este principio al español (una lengua pro-drop), por interpretarlo como una lengua que no permite elidir el sujeto, como las demás L2 que aprenden. Por eso los estudiantes pueden caer en un uso abusivo del sujeto pronominal, cuando no es necesario o no está regido por razones gramaticales, discursivas o comunicativas. Después de haber recibido suficiente input, poco a poco, los aprendices van dejando de utilizar indebidamente los sujetos pronominales. No obstante, la observación es más bien empírica y, por ello, se impone un estudio que investigue la interlengua y las interferencias del francés o del inglés como lenguas extranjeras, por un lado, y el español como lengua extranjera en el caso de los aprendices rumanos.

Según Perales y Portillo Mayorga ${ }^{11}$, otra posible explicación del empleo erróneo de los sujetos pronominales (explícitos) es el tipo de ejercicios que piden al aprendiz completar con la forma adecuada del verbo, proporcionando al mismo tiempo los pronombres sujetos para aclarar el contexto morfosintáctico:

$$
\text { él }
$$
(cantar)

nosotros (comer)

Este método busca reforzar la adquisición de la flexión verbal y la concordancia, pero para que el aprendiz no interprete como obligatoria la presencia del pronombre o del sujeto en español, una solución será escribir los pronombres en función de sujeto entre paréntesis, al lado del verbo, o entre corchetes, así:

a.

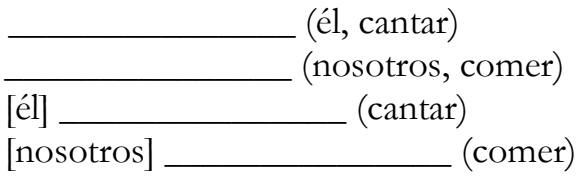

${ }^{11}$ Idem. 
De esta manera, se puede evitar que el aprendiz interprete el pronombre como obligatorio, como parte del enunciado y no sólo como indicación de ejercicio.

\section{Propuestas didácticas}

A continuación, proponemos varios tipos de actividades que podrían facilitar el aprendizaje del sujeto nulo en español. En primer lugar, opinamos que los ejercicios de discriminación serían bastante útiles. Se les puede pedir a los aprendices elegir entre el sujeto pronominal y el sujeto nulo en varios contextos comunicativos. Hay que mencionar a los estudiantes que los sujetos son correferenciales y no se refieren a otras entidades. En (9), Juan y él tienen la misma referencia en la realidad extralingüística.

(9) Juan no se siente bien, porque él / Ø ha comido demasiado.

María ha dicho que ella / Ø viene mañana.

Otro tipo de ejercicio es completar con el pronombre sujeto siempre y cuando sea necesario. De esta manera, los alumnos deciden si el sujeto debería emplearse o no, analizando el contexto situacional.

También, puede haber casos en que la presencia del sujeto marca una diferencia semántica, es decir, se refiere a dos entidades distintas. A modo de ejemplo, en las siguientes oraciones hay una diferencia. En (10a), los sujetos de los dos predicados (piensa y toma) son correferenciales, ambos de refieren al sujeto lexicalizado mediante el sintagma nominal la mayoría de la gente. Por otro lado, en (10b) el sujeto pronominal ella no es correferencial con la mayoría de la gente.

(10) a. La mayoría de la gente i piensa que toma decisiones por sí misma.

b. La mayoría de la gente $e_{i}$ piensa que ella $a_{j}$ toma decisiones por sí misma.

La aproximación contrastiva podría resultar útil en el proceso de aprendizaje de los sujetos nulos y pronominales en español. Los profesores pueden insistir en las similitudes que existen entre el rumano y el español en este ámbito. Las traducciones del rumano al español o al revés ponen de relieve las similitudes entre L1 (el rumano, en nuestro caso) y L2 (el español). La comparación de los casos en que el sujeto se omite en las dos lenguas o se emplea obligatoriamente.

\section{Conclusiones y observaciones finales}

Se puede concluir que, en general, además de las competencias comunicativas y comprensivas, la sintaxis desempeña un papel esencial en el desarrollo de las competencias lingüísticas del usuario de una L2. Por eso, los métodos de enseñanzaaprendizaje tendrán que incluir explicaciones claras y un número suficiente de ejercicios concernientes al uso de los sujetos tácitos y explícitos en español. Más concretamente, entre los tipos de ejercicios posibles se pueden incluir la corrección de oraciones, los 
El uso de los sujetos pronominales explícitos y nulos por los aprendices de ELE

juicios de gramaticalidad, ejercicios de relleno con el sujeto pronominal siempre y cuando haga falta, traducciones, etc.

Es más, hemos observado a la luz de los datos escritos que la frecuencia de los errores que conciernen al uso del sujeto disminuye con el nivel de conocimientos. En esto contribuye no sólo la inmersión en el input, pero también el hecho de que el rumano (la lengua materna de los aprendices) es una lengua de sujeto nulo. A esta conclusión, de que el parámetro del sujeto nulo se aprende bastante rápido y de que sólo en la interlengua inicial dichos errores son más frecuentes, han llegado otros estudiosos. Por consiguiente, después de aprender utilizar correctamente los sujetos (explícitos pronominales vs nulos), es necesario, especialmente en niveles avanzados (C1-C2) reforzar la distinción entre ellos en cuanto a sus propiedades referenciales.

Aunque tanto el español, como el rumano son lenguas pro drop, opinamos que este rasgo común no funciona en el caso de los principiantes, dado que los aprendices todavía están influidos por el carácter pro de los otros idiomas estudiados (el inglés o el francés). Se podría explicar como un caso de interferencia lingüística y ésta podría ser la hipótesis para otras investigaciones en el ámbito del aprendizaje del sujeto español. 